\title{
Experimental Validation of MIMO Multiuser Detection for UMTS High-Speed Downlink Packet Access
}

\author{
Dragan Samardzija \\ Bell Labs (Lucent Technologies) \\ Holmdel, NJ 07733 \\ Email: dragan@lucent.com
}

\author{
Angel Lozano \\ Bell Labs (Lucent Technologies) \\ Holmdel, NJ 07733 \\ Email: aloz@lucent.com
}

\author{
Constantinos Papadias \\ Bell Labs (Lucent Technologies) \\ Holmdel, NJ 07733 \\ Email: papadias@lucent.com
}

\begin{abstract}
In single-user MIMO communication, the firstorder throughput scaling is determined by the smallest of the number of transmit and receive antennas. This typically renders terminals the constraining bottleneck. In a multiuser downlink, this bottleneck can be bypassed by having the base station communicate with multiple terminals simultaneously, whereby the receive antennas at those terminals are effectively pooled in terms of throughput scaling. This, however, requires that the base have instantaneous channel information. Without such information, the structure and statistics of the channel can be exploited to form multiple simultaneous beams towards the various users, but these beams are in general mutually interfering.

Focusing on UMTS HSDPA (high-speed downlink packet access), this paper proposes the use of multiuser detection to discriminate the signals conveyed over interfering beams. This approach is formulated and experimentally evaluated on an HSDPA MIMO testbed that involves a commercial base station, multi-antenna terminals, and custom ASICs.
\end{abstract}

\section{INTRODUCTION}

MIMO (multi-input multi-output) schemes utilizing multiple transmit and receive antennas are posed to be a major ingredient in the evolutionary process of wireless communication. Widely recognized features associated with MIMO are: spatial diversity, signal enhancements, interference mitigation and spatial multiplexing. The latter, in particular, has driven a lot of the research over the last decade, ever since it was shown in [1], [2] that-in rich-scattering conditions-the ergodic capacity (in bits/s/Hz) of a MIMO link as function of the average SNR (signal-to-noise ratio) behaves as

$$
C(\mathrm{SNR})=\min (M, N) \log _{2} \mathrm{SNR}+O(1)
$$

where $M$ and $N$ denote the number of transmit and receive antennas, respectively. This linear scaling with the number of antennas turns out to hold in very general channel conditions [3], [4] and thus it represents a powerful means to achieve high spectral utilization provided that antenna arrays can be effectively deployed.

In actual wireless systems, of course, links do not operate in isolation: each base station must actively communicate with a plurality of users and thus a number of MIMO links have to coexist. The behavior expressed by (1) can be immediately translated onto a multiuser environment by partitioning either time or frequency onto orthogonal sets, each of which is assigned to a particular user link. Focusing on the downlink, where $M$ indicates the number of transmit antennas at the base station while $N$ represents the number of receive antennas at the terminal, such orthogonal multiplexing incurs only a small loss in capacity if $N \gg M$. Unfortunately, the small size and cost sensitivity of mobile terminals precludes the deployment of a large number of antennas thereon and hence the most likely scenario for mobile systems corresponds to $M \geq N$. (In some cases, $N$ may be tightly restricted to equal 1.) In these conditions, orthogonal multiplexing severely constrains the capacity.

Without the constraint of time/frequency multiplexing, a downlink with $M$ antennas at the base and $N$ antennas at each of $K$ users can yield a sum capacity that behaves as [5]

$$
C(\mathrm{sNR})=\min (M, K N) \log _{2} \mathrm{sNR}+O(1)
$$

whereby the tight restrictions on $N$ become immaterial and the burden of limiting the capacity shifts to the base station, where $M$ can be more easily scaled. Unfortunately, achieving (2) requires that the base station have accurate and instantaneous information about the state of the fading channel between each of its antennas and those at each of the mobiles. This represents a total of $M \times K \times N$ time-varying complex quantities, whose instantaneous tracking by the base station is not a viable option in frequency-duplexed systems.

Without instantaneous channel state information at the transmitter, simultaneous transmission to multiple users becomes challenging. In these conditions, interestingly, antenna correlation-often detrimental in MIMO — facilitates the formation of beams that can be directed to individual users providing partial isolation between their simultaneous transmissions. Moreover, this approach (already recognized and incorporated into the UMTS evolutionary process [6], [7]) results in simple receiver structures. The degree of user isolation that can be attained through the composition of beams, however, is directly determined by the location of the users in the cell and by the characteristics of their propagation channels. Unless $K \ll M$, every beam will often illuminate 
users other than the intended one resulting in significant levels of multiuser interference.

In this paper, we formulate and experimentally evaluate a scheme that provides resilience to strong multiuser interference when multiple beams are simultaneously active. The cornerstone of this scheme is the recognition that wellknown MUD (multiuser techniques) [8], developed originally for CDMA (code-division multiple access), can be applied to the mitigation and removal of spatial interference. This represents, to some extent, an abandonment of the idea of simple and basically passive terminals and an embracing of the concept of smart terminals that actively participate in the task of discriminating transmissions to the different users. This conceptual shift is grounded on the rapid improvement in processing power that stems from Moore's law.

In the remainder of the paper, we describe the MIMOMUD scheme and we quantify its benefits through a series of experiments executed on a testbed that involves a commercial base station equipped with multiple antennas, terminals also equipped with multiple antennas, and specially designed MIMO ASICs (application specific integrated circuits). To the best of our knowledge, these are the first such reported experiments.

\section{MIMO-MUD: FORMULATION}

Although not a requirement when MUD is used, we shall limit the number of active users to be $K \leq M$, which allows for the generation of beams that are orthogonal in origin. Larger numbers of users can of course be accommodated via time/frequency-multiplexing.

The baseband complex linear model describing the communication between the base station and the $k$-th terminal, $k \in\{1, \ldots, K\}$, is

$$
\mathbf{y}_{k}=\mathbf{H}_{k} \mathbf{x}+\mathbf{n}_{k}
$$

where $\mathbf{y}_{k}$ is the $(N \times 1)$ vector received by terminal $k, \mathbf{n}_{k}$ is the corresponding additive white Gaussian noise vector with one-sided spectral density

$$
N_{0}=\frac{E\left[\|\mathbf{n}\|^{2}\right]}{N}
$$

and $\mathbf{H}_{k}$ is the $(N \times M)$ channel random matrix whose $(i, j)$-th entry represents the transfer coefficient ${ }^{1}$ between the $j$-th base transmit antenna and the $i$-th receive antenna at terminal $k$. In turn, $\mathbf{x}$ is the $(M \times 1)$ transmit vector, common to all users and structured as

$$
\mathbf{x}=\sum_{\ell=1}^{K} \mathbf{w}_{\ell} s_{\ell}
$$

where $s_{\ell}$ is the information-bearing signal intended for terminal $\ell$ while the vector $\mathbf{w}_{\ell}$ contains the set of deterministic coefficients that, applied to each of the transmit antennas, generate the corresponding beam. Without loss of generality,

\footnotetext{
${ }^{1}$ In order to focus on the spatial processing aspects, the channel fading is modelled as frequency-flat. The formulation, nonetheless, can be extended to frequency-selective fading.
}

the $\mathbf{w}_{\ell}$ 's are chosen such that $\left\|\mathbf{w}_{\ell}\right\|=1, \forall \ell$, and the power radiated for user $\ell$ is then $P_{\ell}=E\left[\left|s_{\ell}\right|^{2}\right]$. It is important to point out that, without instantaneous channel information at the transmitter, the coefficients in the set of vectors $\mathbf{w}_{\ell}$ cannot depend on the random matrices, but only on their distributions.

From the standpoint of user $k$, we can conveniently rearrange (3) and (4) as

$$
\mathbf{y}_{k}=\underbrace{\mathbf{H}_{k} \mathbf{w}_{k} s_{k}}_{\begin{array}{c}
\text { signal intended } \\
\text { for terminal } k
\end{array}}+\underbrace{\sum_{\ell \neq k} \mathbf{H}_{k} \mathbf{w}_{\ell} s_{\ell}}_{\text {interference }}+\underbrace{\mathbf{n}_{k}}_{\text {noise }}
$$

where the interference corresponds to the mutually independent signals that are being beamed towards terminals other than $k$, orthogonal in origin but-in general-not upon reception because of the random matrix $\mathbf{H}_{k}$ whose realization is unknown to the transmitter. The realization of $\mathbf{H}_{k}$, in contrast, is considered known to the receiver, which may estimate if e.g. each individual beam is associated with a unique pilot. (Multiple secondary pilots are already supported in UMTS [7] and are expected to be equally available in future system designs.) More specifically, this enables receiver $k$ to estimate the effective channels $\mathbf{H}_{k} \mathbf{w}_{\ell}$ for $\ell \in\{1, \ldots, K\}$.

There are several manners in which the presence of the interference can be addressed:

- The simplest approach is to ignore the interference by matching the receiver at terminal $k$ to the effective channel for its desired signal generating the decision statistic $\left(\mathbf{w}_{k}^{\dagger} \mathbf{H}_{k}^{\dagger} \mathbf{y}_{k}\right)$, which exhibits an average signalto-interference and noise ratio [8]

$$
\operatorname{SINR}_{k}=E\left[\frac{P_{k} \mathbf{w}_{k}^{\dagger} \mathbf{H}_{k}^{\dagger} \mathbf{H}_{k} \mathbf{w}_{k}}{N_{0}+\frac{\mathbf{w}_{k}^{\dagger} \mathbf{H}_{k}^{\dagger} \mathbf{H}_{k}\left(\sum_{\ell \neq k} P_{\ell} \mathbf{w}_{\ell} \mathbf{w}_{\ell}^{\dagger}\right) \mathbf{H}_{k}^{\dagger} \mathbf{H}_{k} \mathbf{w}_{k}}{\mathbf{w}_{k}^{\dagger} \mathbf{H}_{k}^{\dagger} \mathbf{H}_{k} \mathbf{w}_{k}}}\right]
$$

which depends strongly on the structure of $\mathbf{H}_{k}$. We shall use this SUMF (single-user matched filter) receiver as a baseline for later comparisons.

- A more robust approach consists of mitigating the interference through MMSE (minimum mean-square error) linear processing, which exploits the information provided by the conditional interference covariance

$$
\boldsymbol{\Phi}=N_{0} \mathbf{I}+\sum_{\ell \neq k} P_{\ell} \mathbf{H}_{k} \mathbf{w}_{\ell} \mathbf{w}_{\ell}^{\dagger} \mathbf{H}_{k}^{\dagger}
$$

The resulting average SINR at terminal $k$ is [8]

$$
\operatorname{SINR}_{k}=P_{k} \mathbf{w}_{k}^{\dagger} E\left[\mathbf{H}_{k}^{\dagger} \boldsymbol{\Phi}^{-1} \mathbf{H}_{k}\right] \mathbf{w}_{k}
$$

which must lie within

$$
\frac{P_{k}}{\sum_{\ell \neq k} P_{\ell}} \leq \operatorname{SINR}_{k} \leq \frac{P_{k} \mathbf{w}_{k}^{\dagger} E\left[\mathbf{H}_{k}^{\dagger} \mathbf{H}_{k}\right] \mathbf{w}_{k}}{N_{0}}
$$

The lower bound in (7) corresponds to an interferencelimited situation with $\mathbf{H}_{k}$ having independent entries, in which case the use of beams provides no significant advantage over time/frequency multiplexing, whereas the 
upper bound corresponds to a highly structured channel wherein terminal $k$ receives no interference from any of the beams directed to other users.

- The most ambitious approach, and the one embraced in the remainder of the paper, is based on the joint detection of the signals transmitted on all beams, of which only the intended one is decoded and passed on to the higher layers while the remaining ones are simply discarded. In this case, the average SNR per receive antenna at terminal $k$ is simply

$$
\mathrm{SNR}_{k}=\frac{\sum_{\ell} \mathbf{w}_{\ell}^{\dagger} E\left[\mathbf{H}_{\ell}^{\dagger} \mathbf{H}_{k}\right] \mathbf{w}_{k}}{N_{0} N}
$$

More specifically, the MIMO MUD solution that we propose relies on terminal $k$ using its knowledge of $\mathbf{H}_{k} \mathbf{w}_{\ell} \forall \ell$ to perform ML (maximum likelihood) detection as

$$
\left\{\hat{s}_{1}, \ldots, \hat{s}_{K}\right\}=\arg \min \left\|\mathbf{y}_{k}-\mathbf{H}_{k} \sum_{\ell} \mathbf{w}_{\ell} \hat{s}_{\ell}\right\|^{2}
$$

where $\hat{s}_{k}$ is the estimate of the signal $s_{k}$, retained and processed, while $\hat{s}_{\ell}, \ell \neq k$, are the signals intended for other users, discarded after detection.

\section{UMTS High-Speed Downlink Packet Access MIMO TESTBED}

\section{A. High-Speed Downlink Packet Access}

For delay-tolerant data traffic, upcoming releases of UMTS will allocate a fraction of the power and the code space to HSDPA (high-speed downlink packet access), whose main features are:

- Time multiplexing. Users are time-multiplexed in short frames.

- Multicode signaling. The entire HSDPA code space is assigned to the active user. Thus, the signal consists of a superposition of orthogonal codes.

- No power control. Power control is disabled.

- Link adaptation. The transmit rate is adapted based on feedback from the terminals.

With the incorporation of MIMO, the possibility of having multiple simultaneously active users on separate beams is being considered. It is in this context that the use of MIMOMUD becomes alluring. In the remaining, we validate this idea using an HSDPA-compliant testbed implemented with support for MIMO (4 antennas at the base and 4 antennas at each terminal [9]). The carrier frequency is $2.1 \mathrm{GHz}$ with the regular UMTS bandwidth of $5 \mathrm{MHz}$.

\section{B. Transmitter Implementation}

At the base, omnidirectional vertically polarized $1 / 4$ dipole antennas are set 4 wavelengths apart along a line at a height of about $3 \mathrm{~m}$.

The HSDPA transmitter is mounted on a prototype Lucent base station (OneBTS ${ }^{\mathrm{TM}}$, Fig. 1). A mezzanine board is used to implement the HSDPA physical and MAC layers. The rest of the base station (including RF front end, backplane and network interface) is also used. A FPGA (field programmable gate array) is used to implement the multi-antenna HSDPA physical layer transmitter. Specifically, (i) scrambling and spreading, (ii) modulation, (iii) pilot generation, (iv) channel segmentation, (v) turbo encoding and rate matching, (vi) CRC generation and auxiliary functions. A Xilinx Virtex2 6000 is used with the above functional blocks consuming around $30 \%$ of the FPGA resources.

To support multiple users, the MAC layers are implemented on a processor platform that performs the user scheduling and Hybrid-ARQ. The standard HSDPA specification is retained at the MAC layer and thus only the physical layer is aware of the presence of MIMO.

\section{Receiver Implementation}

The terminal antennas are low-profile bow-tie printed dipoles with alternating $45^{\circ}$ polarizations occupying vertexes of an $8.2^{\prime \prime} \times 5.2^{\prime \prime}$ rectangle (Fig. 2) with the entire array fitting on the back of a laptop. The prototype HSDPA multi-antenna receiver is realized on 3 interconnected printed circuit cards.

Card 1 implements the analog RF front-end outputting up to 4 complex baseband signals, each corresponding to a receive antenna. A heterodyne receiver with optional automatic gain control is utilized.

Card 2 contains the basic processing elements of the multiantenna receiver, (i) MIMO-MUD ASIC [10] and (ii) turbo decoder ASIC [11]. After the AD (analog-to-digital) conversion, the received signal is sent to the MIMO-MUD ASIC which, in turn, outputs LLRs (log-likelihood ratios) that are then fed to the rate dematcher on Card 3. After dematching, the turbo decoder ASIC performs iterative decoding. Each LLR corresponds to 1 channel bit with an 8-bit resolution.

Card 3 holds the FPGA that acts as interconnect matrix between ADs, MIMO-MUD ASIC and turbo decoder ASIC. Further, it executes (i) synchronization, (ii) rate dematching, (iii) channel quality measurements, (iv) CRC check, and numerous auxiliary functions.

\section{MIMO-MUD EXPERIMENTAL RESULTS}

Indoor over-the-air measurements, mostly in static conditions, have been carried out in a laboratory/office environment. The receiver was placed at various locations in the room. QPSK modulation was used with rate- $1 / 2$ coding and 10 length-16 orthogonal codes. The measurements include thermal as well as quantization noise. In order to test the MIMO-MUD concept under the harshest conditions, trivial beams (heavily interfering, no attempt to isolate the users) are employed: each entry of $\mathbf{w}_{\ell}$ is zero except for the $\ell$-th entry, which is set to 1 .

We have measured FERs (frame error rates) with the 2msec time transmission interval specified for HSDPA. Based on the FER and on the 3.84-MHz chip rate, the throughput $T$ is obtained as

$$
T=3.84 M \frac{10}{16}(1-\mathrm{FER})[\mathrm{Mbps}]
$$

This corresponds to a system with ARQ where the frames in error are discarded. 


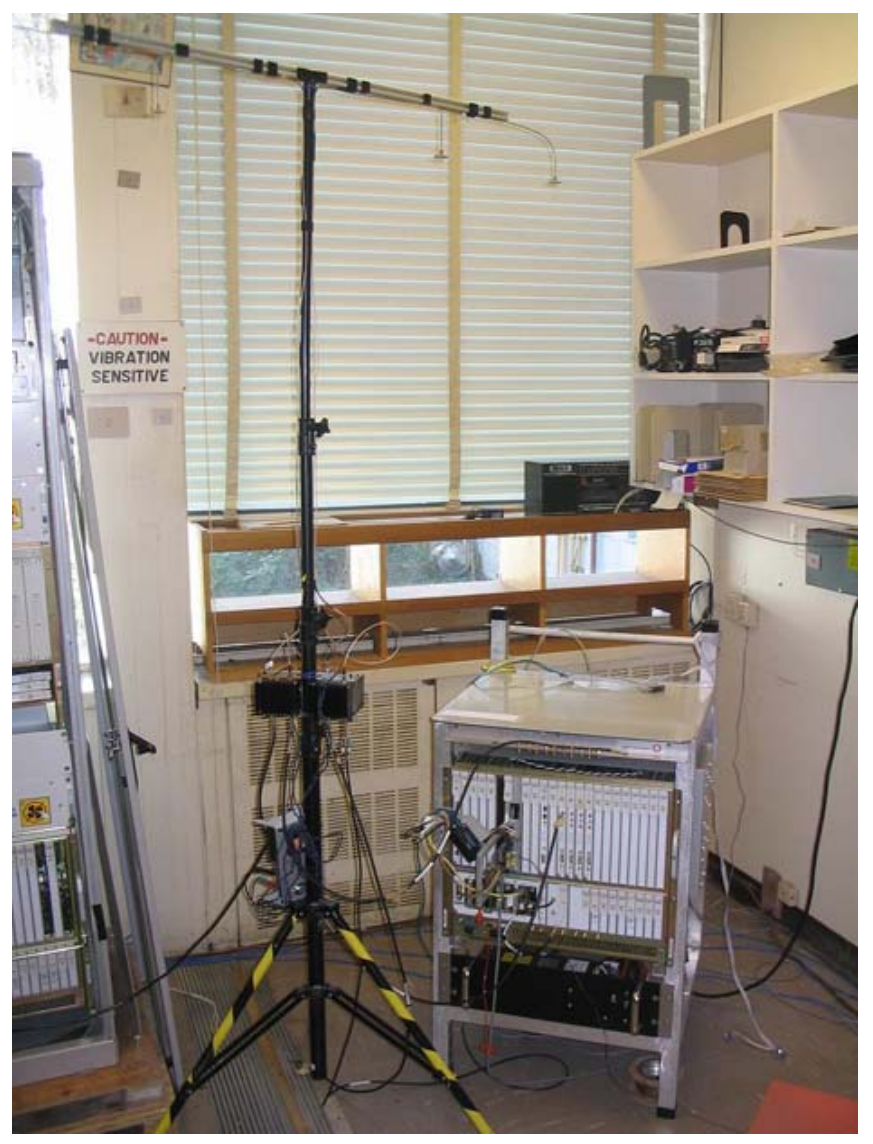

Fig. 1. Multi-antenna HSDPA base station.

Fig. 3 presents the measured CDF (cumulative distribution function) of throughput for a transmit power of $0 \mathrm{dBm}$ over 30 locations. We show, for $M=4$ and $K=4$ terminals each with $N=1$, a comparison of SUMF and MIMO-MUD receivers. Also depicted is the throughput for $K=1$ and $N=1$, for which the SUMF is optimal. Fig. 4 presents the average throughput for different transmit power levels. MIMO-MUD results, at high transmit powers, in an almost 4 -fold increase in average throughput. Fig. 5 presents the average throughput for $N=1,2,3,4$ with $M=4$ and with $0 \mathrm{dBm}$ (solid line) and $10 \mathrm{dBm}$ (dashed line) transmit powers. Fig. 6 presents corresponding results for $M=2$. Although, for higher $N$, the SUMF approaches the MIMO-MUD throughput, this partly is an artifact of the fact that only QPSK is used. With higher order constellations available, we expect the MIMO-MUD advantage to be largely sustained.

\section{CONCLusions}

Multiuser detection is a natural approach to signal detection in multiuser environments. Although much of the developments in this area have been motivated by CDMA, multiuser techniques are equally well suited to the spatial processing that arises with the use of MIMO, where the role of the CDMA spreading sequences is played by the fading coefficients between the various transmit and receive antennas.

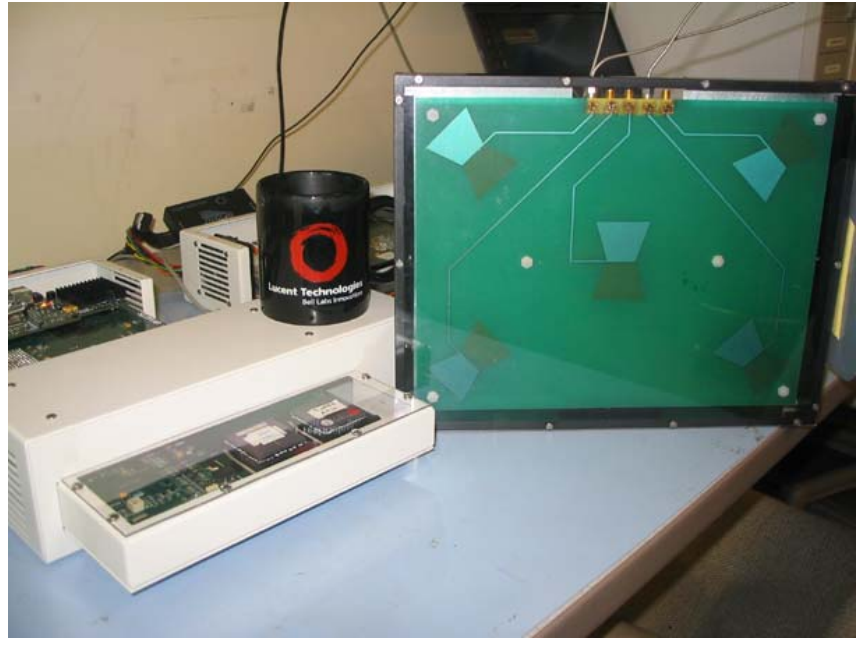

Fig. 2. HSDPA terminal and receive antenna array.

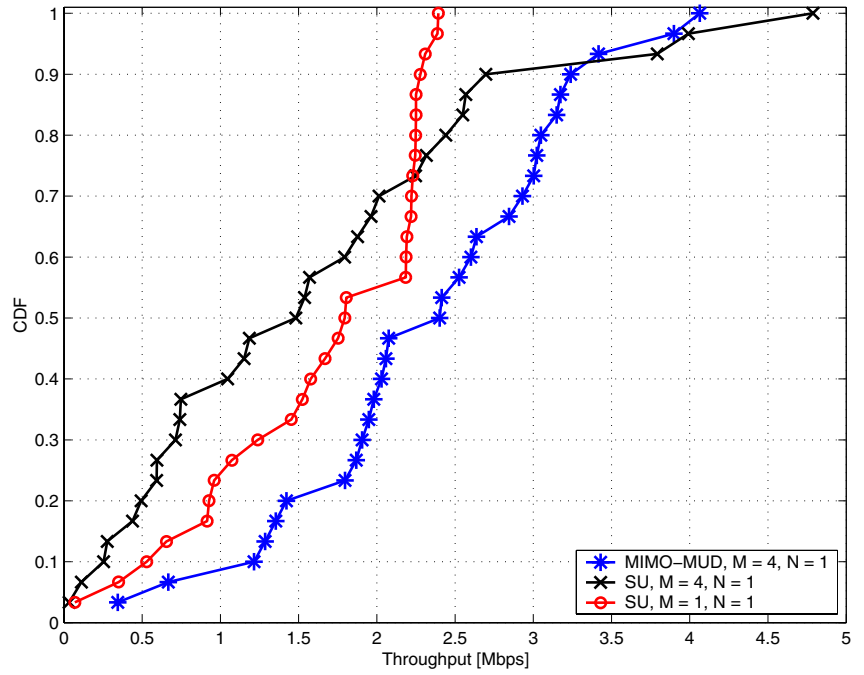

Fig. 3. Measured CDF of throughput for $0 \mathrm{dBm}$ over 30 locations.

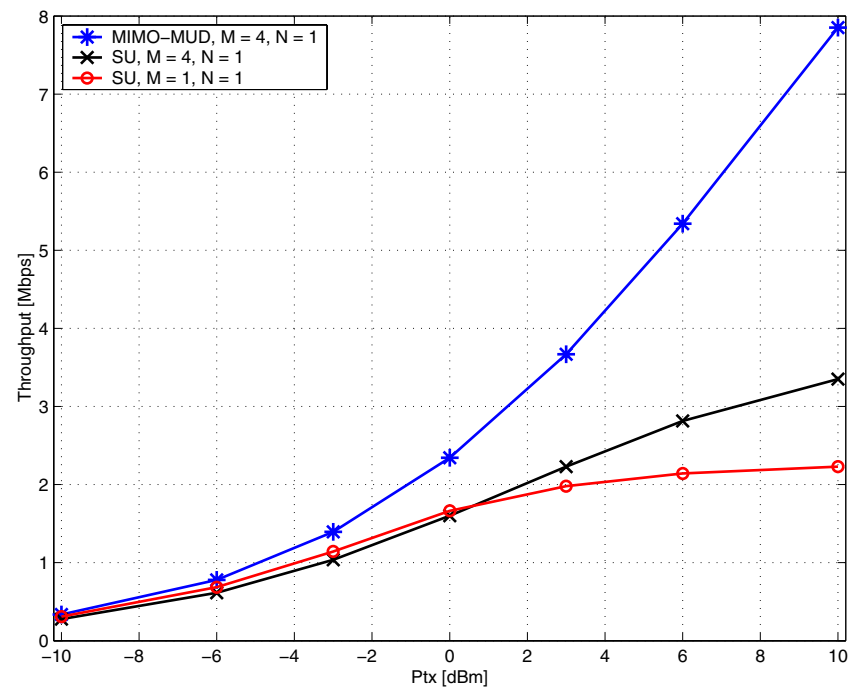

Fig. 4. Measured average throughput vs. transmit power over 30 locations. 


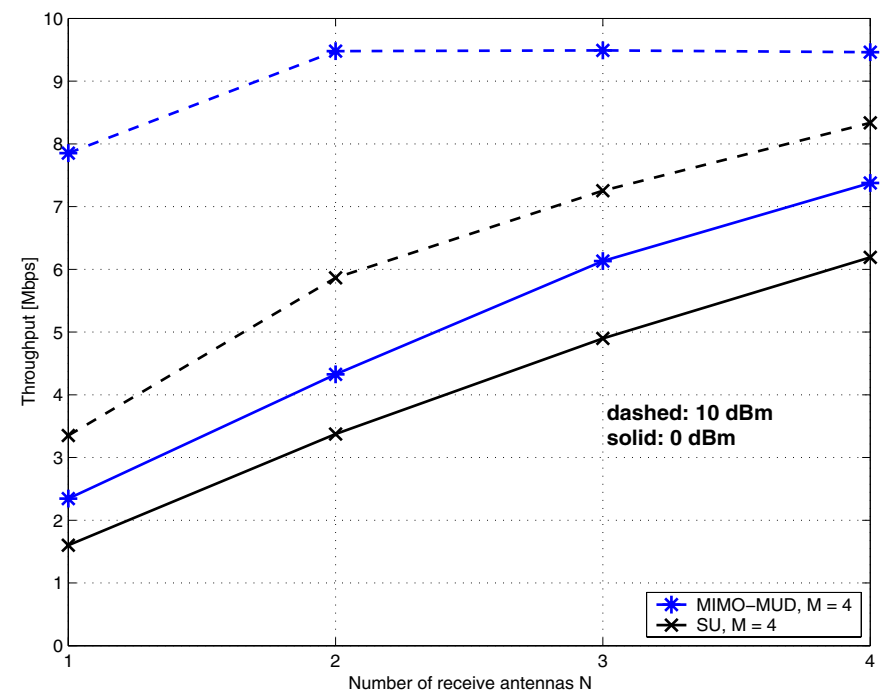

Fig. 5. Measured average throughput vs. $N$ at $0 \mathrm{dBm}$ (solid line) and $10 \mathrm{dBm}$ (dashed line) over 30 locations.

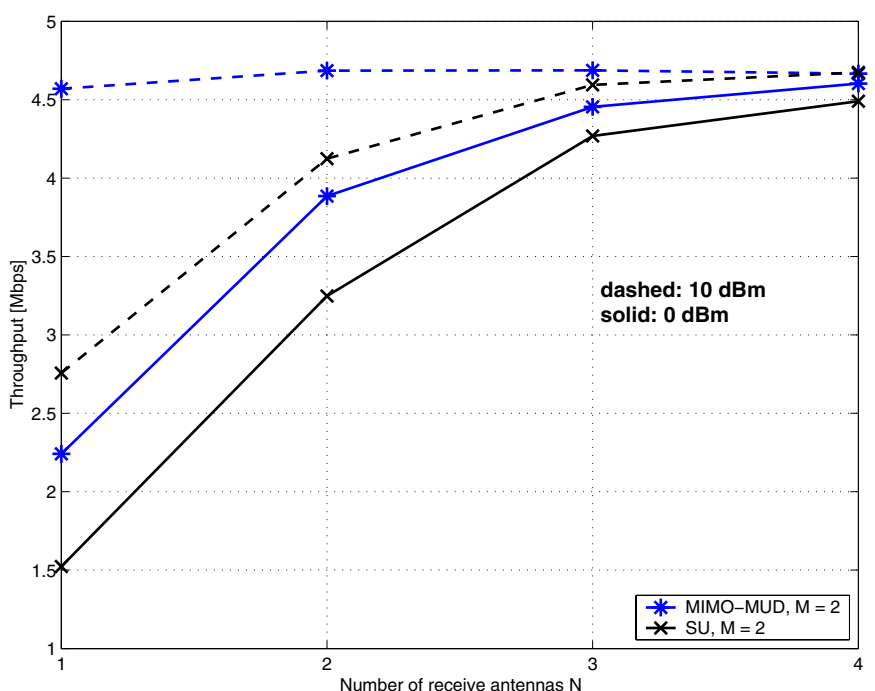

Fig. 6. Measured average throughput vs. $N$ at $0 \mathrm{dBm}$ (solid line) and $10 \mathrm{dBm}$ (dashed line) over 30 locations.

In this paper, we have applied MUD to the detection of mutually interfering downlink beam transmissions aimed at different terminals. Without instantaneous channel state information at the base, these beams cannot be rendered orthogonal at the terminal receivers. Rather than simply enduring their mutual interference, we have proposed to jointly detect the signals transmitted on the intended and unintended beams.

Besides formulating such MIMO MUD reception, we have experimentally validated the approach using a testbed that includes a commercial multi-antenna base station, multi-antenna terminals and custom MIMO ASICs. The results confirm the power of MUD, especially when the number of receive antennas at each terminal does not exceed the number of transmit antennas at the base.

\section{ACKNOWLEDGMENTS}

Part of this work was done under the IST project FITNESS, sponsored and funded by the FP5 European research framework.

\section{REFERENCES}

[1] G. J. Foschini and M. J. Gans, "On the limits of wireless communications in a fading environment when using multiple antennas," Wireless Personal Communications, pp. 315-335, 1998.

[2] I. E. Telatar, "Capacity of multi-antenna Gaussian channels," Eur. Trans. Telecom, vol. 10, pp. 585-595, Nov. 1999.

[3] C. Chuah, D. Tse, J. Kahn, and R. Valenzuela, "Capacity scaling in dual-antenna-array wireless systems," IEEE Trans. on Inform. Theory, vol. 48, no. 3, pp. 637-650, Mar. 2002.

[4] A. M. Tulino, S. Verdú, and A. Lozano, "Capacity of antenna arrays with space, polarization and pattern diversity," Inform. Theory Workshop (ITW'03), Paris, France, Apr. 2003.

[5] G. Caire and S. Shamai, "On achievable throughput of a multiantenna Gaussian broadcast channel," IEEE Trans. on Inform. Theory, vol. 49, no. 7, pp. 1691-1706, July 2003.

[6] K. Pedersen, P. Mogensen, and J. Ramiro-Moreno, "Application and performance of downlink beamforming techniques in UMTS," IEEE Comm. Magazine, pp. 134-143, Oct. 2003.

[7] "Beamforming enhancements," TR 25.887 v. 1.3.0, 3rd Generation Partnership Project, Oct. 2002.

[8] S. Verdú, Multiuser Detection. Cambridge University Press, 1998.

[9] A. Burg, E. Beck, D. Samardzija, M. Rupp, and et al., "Prototype experience for MIMO BLAST over third generation wireless system," IEEE JSAC Special Issue on MIMO Systems and Applications, vol. 21, no. 3, April 2003

[10] D. C. Garrett, L. M. Davis, and G. K. Woodward, "19.2 Mbit/s 4 x 4 BLAST/MIMO detector with soft ML outputs," IEE Electr. Letters, vol. 39, no. 2, pp. 233-235, Jan. 2003.

[11] M. Bickerstaff, L. Davis, C. Thomas, D. Garrett, and C. Nicol, "A 24 $\mathrm{Mb} / \mathrm{s}$ radix-4 turbo decoder," IEEE Intern. Solid-State Circuits Conf., Feb. 2003. 